\title{
Fermion Mass Hierarchy without Flavour Symmetry
}

\author{
Chih-Lung Chou \\ Stanford Linear Accelerator Center, Stanford, CA 94309 \\ Applied Physics Department, Stanford University, Stanford, CA 94309
}

\begin{abstract}
We discuss a supersymmetric grand unified model which has gauge group $\mathrm{SU}(5) \times \mathrm{SU}(5) \times \mathrm{SU}(5)$, with matter fields transforming asymmetrically under different gauge $\mathrm{SU}(5)$ groups. We observe that the gauge structure of the model leads to approximate texture zero structures in fermion mass matrices and a natural hierarchy in the Yukawa couplings. The proton lifetime is estimated to be larger than $10^{34}$ years in this model. As in more conventional supersymmetric GUT models with product gauge groups, this model possesses no tensor fields with rank higher than 2 , so that it might arise from a level 1 string construction.
\end{abstract}

12.10.Kt,10.15.Ff,12.60.Jv,12.10.Dm

Typeset using REVTEX 


\section{INTRODUCTION}

The Standard Model (SM) is now considered to be completely successful in describing the physical world up to the weak scale. However, it requires some 18 parameters which are input by hand to fit the experiment data. Most of these undetermined parameters reflect our lack of understanding of flavour physics. The SM provides no explanation of why there is a mass hierarchy among the fermion masses and no explanation of the CKM angles. It seems that Nature includes some classification which goes beyond the structure of the SM [回]. Thus, in order to solve these puzzles, we have to go beyond the SM. The Minimal Supersymmetric Standard Model (MSSM) [2] has considered as one of the possible extended theories beyond the SM. Despite of its success in providing true gauge coupling unification [3], it also has flavour problems [4] at least as severe as those in the SM. The fermion mass hierarchy is still left unexplained in the MSSM framework. Even worse, new problems such as Flavour Changing Neutral Currents [5] occurs.

Many solutions have been proposed for the flavour problem, either within a supersymmetric framework [6,17] or in non-supersymmetric theories. Most of these attempts assume that some flavour symmetries, gauged or global, exist above the grand unification scale $M_{x}$. The flavour symmetries typically restrict the possible Yukawa coupling terms in the superpotential and provide textures and hierarchy patterns of the fermion mass matrices [7,23, 17]. This idea is often combined with that of grand unification. For example, one can introduce higher rank tensors such as the 126 in the $S O(10)$ grand unified theories (GUT's) [8] and the 45 in the $S U(5)$ GUT's [9] in order to create specific textures in the quark and lepton mass matrices. Theoretically, there is nothing wrong with introducing high rank tensors. However, the affine level 1 constructions in string theory does not allow string-derived GUT's having tensor fields with rank higher than 2 [10]. This result makes the ordinary SUSY $S U(5)$ [11] and $S O(10)$ [12] GUT theories difficult to obtain from the affine level 1 constructions in string theory. In response to this situation, Barbieri et. al. [13 pointed out that extending the GUT gauge group to be $G \times G$, where G could be some GUT groups 
such as the $S U(5)$ or the $S O(10)$ group, makes it possible to construct GUT models which break the product gauge groups down to the SM gauge group without introducing high rank tensor fields. The GUT gauge group in these theories could be broken by fields which carry fundamental and antifundamental representations under two different gauge groups. For examples, the $(5, \overline{5})$ and the $(\overline{5}, 5)$ can break the $S U(5) \times S U(5)$ GUT theories down to the SM gauge group. The same logic applies to theories with gauge group $G \times G \times G$. Furthermore, as pointed out by Barbieri et. al [14], if we choose to have each family of matter fields transforming under different gauge group $G$, then a flavour theory could be constructed without the need for an explicit flavour symmetry group.

In this paper, we follow the idea of using $S U(5) \times S U(5) \times S U(5)$ as the SUSY GUT gauge group. However, instead of symmetrically assigning each family of matter fields $(10+\overline{5})$ to its own gauge group $S U(5)$, we assign the matter fields transforming under these gauge $S U(5)$ 's in an asymmetrical way. In Section 2, we describe our model and suggest a suitable vacuum for those fields which break the GUT gauge group. A $Z_{2} \times Z_{3}$ discrete symmetry at the superheavy scale is introduced to suppress the dangerous operators as well as to obtain a weak-scale $\mu$ value in the model. This gives the full set of assumptions of our construction. In the remainder of the paper, we show that these assumptions lead to many interesting consequences. In Section 3, we derive the fermion mass matrices and demonstrate a mass hierarchy which follows from the gauge structure of our model. We show that our model can account for the observed fermion mass matrices and CKM angles. In Section 4, we discuss

proton decay in this model. The proton lifetime predicted in this model is consistent with the limit set by the SuperKamiokande experiment. In Section 5, we present some conclusions.

\section{THE MODEL}

Our model is based on the SUSY GUT gauge group $S U(5)_{1} \times S U(5)_{2} \times S U(5)_{3}$. We identify the SM gauge group $S U(3)_{C} \times S U(2)_{L} \times U(1)_{Y}$ as lying in a diagonal $S U(5)$ subgroup of above product group. To break the GUT gauge group down to the SM $S U(3)_{C} \times S U(2)_{L}$ 
$\times U(1)_{Y}$, we require the exotic Higgs fields $T_{1}, T_{2}$ and $T_{3}$ in the representations $(1,5, \overline{5})$, $(\overline{5}, 1,5)$ and $(5, \overline{5}, 1)$. We will find it useful to add two more multiplets, $\Sigma$ in the $(1,5, \overline{5})$ and $\bar{\Sigma}$ in the $(1, \overline{5}, 5)$. We assign the three 10 's of $S U(5)$ to the three different $S U(5)$ groups and we associate the 5 and $\overline{5}$ Higgs fields with different groups. However, we assign two $\overline{5}$ matter multiplets to the same $S U(5)$. The complete set of assignment is shown in Table 1. According to the assignment in Table 1, there is already some interesting physics at the level of lower dimension operators. The ordinary $\mu$ term

$$
\mu H \bar{H}
$$

is forbidden from appearing in the fundamental Lagrangian by gauge invariance. The leading contribution to the $\mu$ term potentially comes from high dimension operators in the superpotential and will be analyzed further in the later of this section.

As one can see from the table, this model contains no fields in the adjoint representation, and no fields with rank higher than 2. All of these fields can appear in a string construction with the gauge group realized at the affine level $k=1$ [10]. The breaking of the GUT gauge group can be accomplished by the vacuum expectation values (VEV's) of the fields $T_{1}, T_{2}, T_{3}, \Sigma$ and $\bar{\Sigma}$. The symmetry-breaking ground state could be either by a stablized tree-level superpotential or by effects of a strongly coupled SUSY gauge theory. Here, before discussing an explicit potential, we would like to propose a possible vacuum which can break the gauge group $S U(5)_{1} \times S U(5)_{2} \times S U(5)_{3}$ down to the SM gauge group $S U(3)_{C} \times S U(2)_{L}$ $\times U(1)_{Y}$. We assume that the symmetry is broken in two steps. First, $S U(5)_{1} \times S U(5)_{3}$ is broken to the diagonal subgroups by an expection value of $T_{2}$.

$$
\left\langle T_{2}\right\rangle=\Lambda_{2} \cdot \operatorname{diag}(1,1,1,1,1)
$$

Then the remaining symmetry $S U(5)_{D 31} \times S U(5)_{2}$ is broken to the $S U(3)_{C} \times S U(2)_{L} \times U(1)_{Y}$ by the expection values of $T_{1}$ and $\Sigma$.

$$
\begin{array}{r}
\left\langle T_{1}\right\rangle=\Lambda_{1} \cdot \operatorname{diag}(0,0,0,1,1) \\
\langle\Sigma\rangle=\Omega \cdot \operatorname{diag}(1,1,1,0,0)
\end{array}
$$


Finally, the remaining fields get their expection values along the $S U(3)_{C} \times S U(2)_{L} \times U(1)_{Y}$ direction. Only relatively small hierarchies between these scales are needed to produce large hierarchies in the quark mass matrices. We will show this in Section 3. The complete pattern of VEV's consistent with the symmetry breaking pattern just described is:

$$
\begin{aligned}
\langle\Sigma\rangle=\Omega \cdot \operatorname{diag}(1,1,1,0,0) & \langle\bar{\Sigma}\rangle=\bar{\Omega} \cdot \operatorname{diag}(1,1,1, a, a) \\
\left\langle T_{1}\right\rangle=\Lambda_{1} \cdot \operatorname{diag}(0,0,0,1,1) & \left\langle T_{3}\right\rangle=\Lambda_{3} \cdot \operatorname{diag}(1,1,1, s, s) \\
\left\langle T_{2}\right\rangle & =\Lambda_{2} \cdot \operatorname{diag}(1,1,1,1,1)+\Lambda_{3} \cdot \operatorname{diag}(0,0,0, b, b)
\end{aligned}
$$

Due to the $S U(5)_{1}$ D-term condition, the $\operatorname{VEV}\left\langle T_{2}\right\rangle$ will receive a correction of order $O\left(\Lambda_{3}\right)$. The constants $a, b$ and $s$ are assumed to be nonzero and would be determined by minimizing the potential. We will show below that the zeros in $\Sigma$ and $T_{1}$ can be exact, up to the point where SUSY is spontaneously broken. As in conventional GUT models, we also requuire a discrete symmetry to forbid dangerous operators such as $H \overline{5}_{1}, H \overline{5}_{3}, 10_{3} \overline{5}_{3} \overline{5}_{1}$ and $T_{1} T_{3} H \overline{5}_{2}$ in the tree-level superpotential. Specifically, we assume a $Z_{2}^{\text {matter }} \times Z_{3}$ symmetry

$$
\begin{aligned}
& Z_{2}^{\text {matter }}:\left(10_{1}, 10_{2}, 10_{3}, \overline{5}_{1}, \overline{5}_{2}, \overline{5}_{3}\right) \longrightarrow-1\left(10_{1}, 10_{2}, 10_{3}, \overline{5}_{1}, \overline{5}_{2}, \overline{5}_{3}\right) \\
& Z_{3}:\left(H, \bar{H}, \Sigma, \bar{\Sigma}, 10_{3}\right) \longrightarrow\left(H, \bar{H}, \Sigma, \bar{\Sigma}, 10_{3}\right) \\
&\left(T_{1}, T_{3}, 10_{2}, \overline{5}_{2}\right) \longrightarrow e^{i 2 \pi / 3}\left(T_{1}, T_{3}, 10_{2}, \overline{5}_{2}\right) \\
&\left(T_{2}, 10_{1}, \overline{5}_{1}, \overline{5}_{3}\right) \longrightarrow e^{i 4 \pi / 3}\left(T_{2}, 10_{1}, \overline{5}_{1}, \overline{5}_{3}\right)
\end{aligned}
$$

The dangerous dimension five operators that could make the proton decay too rapidly are also suppressed by the $Z_{3}$ symmetry. We will discuss this in Section 4 . We now discuss the spectra of Higgs masses and the $\mu$ paramater. Applying the $Z_{2}^{\text {matter }} \times Z_{3}$ symmetry, we can easily write all possible leading terms up to dimension 10 level that are bilinear in $H$ and $\bar{H}$.

$$
\begin{aligned}
W_{H \bar{H}} & =\Sigma H \bar{H}\left\{1+\frac{\Sigma \bar{\Sigma}}{M^{2}}+\frac{\Sigma T_{2} T_{3}}{M^{3}}+\frac{(\Sigma \bar{\Sigma})^{2}}{M^{4}}+\frac{\Sigma^{5}+\Sigma^{2} T_{1}^{3}}{M^{5}}+\frac{(\Sigma \bar{\Sigma})\left(\Sigma T_{2} T_{3}\right)}{M^{5}}\right. \\
& \left.+\frac{(\Sigma \bar{\Sigma})^{3}+\left(T_{1} \bar{\Sigma}\right)^{3}+\left(\Sigma T_{2} T_{3}\right)^{2}}{M^{6}}+\sum_{k=0}^{5} \frac{1}{M^{5-k}} \bar{\Sigma}^{k}\left(T_{2} T_{3}\right)^{5-k}\right\}
\end{aligned}
$$




$$
\begin{aligned}
& +T_{1} H \bar{H}\left\{\frac{\left(T_{1} \bar{\Sigma}\right)^{2}}{M^{4}}+\frac{\Sigma^{3} T_{1}^{2}+T_{1}^{5}+T_{3}^{5}+\left(T_{1} \bar{\Sigma}\right)\left(T_{1} T_{2} T_{3}\right)}{M^{5}}\right. \\
& \left.+\frac{\left(T_{1} T_{2} T_{3}\right)^{2}}{M^{6}}\right\}
\end{aligned}
$$

From the vacuum state described in Eq. (5), not all terms in Eq. (7) would have non-zero contributions to the Higgs triplet mass and the $\mu$ value. The Higgs triplets get a superheavy mass $\Omega$ which is shown to be of $O\left(10^{16}\right) \mathrm{GeV}$ in the next section. The leading terms that give $\mu$ a nonzero value are

$$
\mu \approx\left\langle T_{1}\left[\frac{\left(T_{1} \bar{\Sigma}\right)^{2}}{M^{4}}+\frac{\Sigma^{3} T_{1}^{2}+T_{3}^{5}+\left(T_{1} \bar{\Sigma}\right)\left(T_{1} T_{2} T_{3}\right)}{M^{5}}+\frac{\left(T_{1} T_{2} T_{3}\right)^{2}}{M^{6}}\right]\right\rangle .
$$

Eq. (8) is highly suppressed by $1 / M^{4}$. When we estimate the various paramaters in the next section, we will see that $\mu$ obtains a weak-scale $\mu$ value.

It is important to ask whether the pattern of VEV's that we have considered in the Eq. (5) can follow from a tree-level superpotential. There is an example of a superpotential that can lead to this structure which incorporates the constraints of $Z_{2}^{\text {matter }} \times Z_{3}$ symmetry.

$$
\begin{aligned}
W\left(\Sigma, \bar{\Sigma}, T_{1}, T_{2}, T_{3}\right) & =\frac{1}{M^{3}} Y_{1}\left(\Sigma^{3} T_{1}^{2}-\phi_{1}^{5}\right)+\frac{1}{M^{3}} Y_{2}\left(T_{2}^{5}-\phi_{2}^{5}\right)+\frac{1}{M^{3}} Y_{3} \Sigma^{4} T_{1} \\
& +\frac{1}{M^{3}} Y_{4}\left(\Sigma^{2} T_{1}^{3}\right)+\frac{1}{M} Y_{5}\left(\phi_{1}^{3}-\Lambda^{3}\right)+\frac{1}{M} Y_{6}\left(\phi_{2}^{3}-\Lambda_{2}^{3}\right) \\
& +\sum_{i=1}^{6} X_{i} Y_{i}^{2}+Y_{7} \Sigma \bar{\Sigma}+\frac{1}{M} Y_{8} \Sigma T_{2} T_{3}+M X_{7} Y_{7}+M X_{8} Y_{8} \\
& +\frac{A_{1}}{M}(\Sigma \Sigma)^{2}+\frac{B_{1}}{M^{2}}(\Sigma \bar{\Sigma})\left(\Sigma T_{2} T_{3}\right)+\frac{B_{2}}{M^{3}}\left(\Sigma T_{2} T_{3}\right)^{2} \\
& +\sum_{i, j \geq 0}^{3} C_{i j} \frac{\left(T_{1} \bar{\Sigma}\right)^{i}\left(T_{1} T_{2} T_{3}\right)^{j}\left(T_{2}^{5}\right)^{3-i-j}}{M^{12-3 i-2 j}}
\end{aligned}
$$

Here $A_{i}, B_{i}$ and $C_{i j}$ are understood as the unspecified coefficients and $\mathrm{M}$ is the super-high scale. The gauge singlets $\phi_{i}, Y_{i}$ and $X_{i}$ are needed to produce the following constraints

$$
\begin{aligned}
\left\langle\Sigma^{3} T_{1}^{2}\right\rangle & =\Lambda^{5}, & \left\langle T_{2}^{5}\right\rangle & =\Lambda_{2}^{5} \\
\left\langle\Sigma^{4} T_{1}\right\rangle & =0, & \left\langle\Sigma^{2} T_{1}^{3}\right\rangle & =0 .
\end{aligned}
$$

These lead to the zero texture patterns in the VEV's of $\Sigma$ and $T_{1}$. The F-term conditions from the superpotential (9) as well as the D-term conditions of the GUT gauge groups would 
determine the possible vacua of this model. The $S U(5)_{3}$ D-term as well as the $S U(5)_{2} \mathrm{D}$ term conditions could force the scales $\Lambda_{1}$ and $\Omega$ to have approximately equal value $\Lambda_{1} \approx \Omega$ if $\Lambda_{1}$ is much larger than $\Lambda_{3}$ and $\bar{\Omega}$. Typically, solving for the minima of a potential would give rise to many discretely degenerate vacua. This is generic to most SUSY GUT theories [14,13,22 if a tree-level superpotential is responsible for breaking the GUT gauge group.

The above Higgs triplet-doublet splitting mechanism is similiar to the sliding-singlet mechanism 15. The Higgs triplets and doublets split when the field $\Sigma$ get superheavy VEV's in its $S U(3)$ block, while keeping vanishing VEV's in its $S U(2)$ block. This description applies to the theory before supersymmetry breaking. It is a well-known difficulty of the sliding-singlet mechanism that SUSY breaking effects could bring corrections to the VEV of $\Sigma$ and may destory the gauge hierarchy [16]. We will now argue that this is not a problem in our model.

To be explicit, the problem resides in [16] is that the low energy effective singlet field $\Sigma_{s}$ that comes from the field $\Sigma$ couples to the superheavy heavy triplets in $H$ and $\bar{H}$. If we turn on SUSY breaking effects, this would give rise to one-loop tadpole graphs which induce the following two terms in the low energy effective theory.

$$
\begin{gathered}
c_{1} m_{g}^{2} M_{G} \Sigma_{s}+\text { h.c. } \\
c_{2} m_{g} M_{G} F_{\Sigma_{s}}+\text { h.c. }
\end{gathered}
$$

Here $m_{g}$ represents the gaugino mass and $M_{G}$ represents the GUT mass scale. These terms shift the VEV's of $\Sigma$ and $T_{1}$. Adding Eq. (12) to the effective theory, the piece of the potential that could shift the VEV's of $\Sigma$ in its $S U(2)$ block is given by

$$
\begin{aligned}
V & =\left(|\langle H\rangle|^{2}+|\langle\bar{H}\rangle|^{2}\right)|\langle\Sigma\rangle|^{2}+\left|\left\langle\frac{1}{M^{3}} \Sigma^{4} T_{1}\right\rangle\right|^{2}+\left|\left\langle\frac{1}{M^{3}}\left(\Sigma^{3} T_{1}^{2}-\Lambda^{5}\right)\right\rangle\right|^{2} \\
& +\left|\left\langle\frac{\Sigma^{2} T_{1}^{3}}{M^{3}}\right\rangle\right|+\left|\left\langle\Sigma \bar{\Sigma}+M X_{7}\right\rangle\right|^{2}+\left|\left\langle\frac{1}{M} \Sigma T_{2} T_{3}+M X_{8}\right\rangle\right|^{2}+c_{1} m_{g}^{2} M_{G} \Sigma_{s} \\
& +\cdots
\end{aligned}
$$

Inserting $\Sigma \longrightarrow \Sigma+\Delta \Sigma$ into Eq. (14), we find the possible shift of the $S U(2)$ VEV's put 


$$
\Delta \Sigma_{2} \lesssim \frac{c_{1} m_{g}^{2} M_{G}}{2\left|\frac{4}{M^{3}} \Sigma^{3} T_{1}\right|^{2}} \sim O\left(10^{2}\right) \mathrm{GeV}
$$

For the same reason, the VEV's in the $S U(3)$ block of the field $T_{1}$ could also receive an

order $10^{2} \mathrm{GeV}$ correction. As one can see from Eq. (15), the shift of the VEV $\langle\Sigma\rangle$ in its $S U(2)$ block is bounded and would not destroy the gauge hierarchy. The same strategy can be applied to the term in Eq. (13). After eliminating the auxiliary field $F_{\Sigma_{s}}$, this term gives a potential of the form

$$
\left|Y_{7} \bar{\Sigma}+\frac{Y_{8} T_{2} T_{3}}{M}+\frac{4 Y_{3} \Sigma^{3} T_{1}}{M^{3}}+\frac{3 Y_{1} \Sigma^{2} T_{1}^{2}}{M^{3}}+\frac{2 Y_{4} \Sigma T_{1}^{3}}{M^{3}}+H \bar{H}+c_{2} m_{g} M_{G}+\cdots\right|^{2}
$$

This modification can shift the VEV's of the singlets $Y_{1}, Y_{3}$ by an amount of order $10^{9} \mathrm{Gev}$

or shift the VEV's of the singlets $Y_{7}$ and $Y_{8}$ by an amount of order of $10^{4}$ and $10^{5} \mathrm{GeV}$; this gives a small correction to the potential which is consistent with the hierarchy.

In this section, by extending the GUT group from the commonly used $S U(5)$ group to $S U(5)_{1} \times S U(5)_{2} \times S U(5)_{3}$, we are allowed to solve the Higgs triplet-doublet splitting problem and give $\mu$ a weak-scale value. It seems that having the $\mathrm{H}$ and $\bar{H}$ transform under different $S U(5)$ gauge groups gives a natural mechanism for solving these problems. Thus it is wellmotivated to introduce product groups like $S U(5) \times S U(5)$ or the $S U(5) \times S U(5) \times S U(5)$ group as potential SUSY GUT gauge groups.

\section{THE FERMION MASS MATRICES}

Now we examine the structure of the Yukuwa couplings in our model. Just as we constructed the terms bilinear in Higgs fields, it is straightforward to write the terms bilinear in quark and lepton fields. For the up quark masses, there are terms that apply $H 10_{i} 10_{j}$ to various combinations of the GUT-level Higgs fields.

$$
\begin{aligned}
W_{u p} & =H\left\{10_{3} 10_{3}+\frac{T_{1}}{M} 10_{2} 10_{2}+\frac{T_{2}^{2}}{M^{2}} 10_{3} 10_{1}+\frac{T_{1} T_{3}}{M^{2}} 10_{1} 10_{1}+\right. \\
& \left.+10_{3} 10_{2}\left(\frac{T_{1}^{2} \Sigma}{M^{3}}\right)+10_{2} 10_{1}\left(\frac{T_{1}^{2} T_{2}^{2} \Sigma}{M^{5}}\right)+\cdots\right\}
\end{aligned}
$$


In the above superpotential $W_{u p}$, we list only the leading terms to various combinations bilinear in fields $10_{i}$. The omitted terms in Eq. (17) represent possible next to leading order combinations. For the down quark and lepton masses, we find terms that include $\bar{H} 10_{i} \overline{5}_{j}$ contracted with various combinations of the GUT-level Higgs fields.

$$
\begin{aligned}
W_{\text {down-lepton }} & =\bar{H}\left\{\frac{T_{1}}{M} 10_{3} \overline{5}_{3}+\left(\frac{T_{1}(\Sigma \bar{\Sigma})}{M^{3}}+\frac{T_{1}\left(\Sigma T_{2} T_{3}\right)}{M^{4}}\right) 10_{3} \overline{5}_{1}\right. \\
& \left.+10_{3} \overline{5}_{2}\left[\frac{\left(T_{1} \bar{\Sigma}\right)\left(\Sigma^{2} T_{3}\right)}{M^{5}}\right)\right]+\frac{T_{3} T_{2}+\bar{\Sigma}}{M^{2}} 10_{2} \overline{5}_{3}+\frac{T_{3}}{M} 10_{2} \overline{5}_{2} \\
& +\left[\frac{T_{3} T_{2}}{M^{2}}+\frac{\bar{\Sigma}}{M}\right] 10_{2} \overline{5}_{1}+\frac{T_{1} T_{2}^{2}}{M^{3}} 10_{1} \overline{5}_{3}+\frac{T_{2} T_{1}}{M^{2}} 10_{1} \overline{5}_{2}+\frac{T_{1} T_{2}^{2}}{M^{3}} 10_{1} \overline{5}_{1} \\
& +\cdots\}
\end{aligned}
$$

We have defined the two matter fields $5_{1}$ and $5_{3}$, which have the same gauge and $Z_{2}^{\text {matter }} \times Z_{3}$ quantum numbers, so that the first term of Eq. (18) contains only $5_{3}$, and $5_{1}$ is the orthogonal linear combination. We have ignored all the coefficients that could appear in front of each coupling term in Eq.s (17) and (18). Terms such as $T_{2}^{4} 10_{1} 10_{1} H / M^{4}$ and $\bar{\Sigma}^{4} 10_{3} \overline{5}_{3} \bar{H} / M^{4}$ in the superpotentials $W_{u p}$ and $W_{\text {down-lepton }}$ are not listed because they are the higher-order contributions to the entries of the fermion mass matrices. We will see this point much clearly in the later discussion of this section. However, as we will see in Section 4, the term $\left(T_{2}^{4} / M^{4}\right) 10_{1} 10_{1} H$ cannot be ignored in the discussion of the proton decay in the model. As is typical in GUT theories based on $S U(5)$ unification [11, the up-type fermion masses are seen to be unrelated to the down- and lepton-type fermion masses.

According to Eq.s (17) and (18), only the top quark will receive a weak-scale mass. All other fermion masses arise from nonrenormalizable couplings and thus are suppressed by powers of $1 / M$. These powers, together with the various VEV's in Eq. (5), lead to a hierarchy of Yukawa couplings. To exhibit this hierarchy, define the small paramaters $\rho=\Omega / M$, $\bar{\rho}=\bar{\Omega} / M, \xi_{1}=\Lambda_{1} / M, \xi_{2}=\Lambda_{2} / M$ and $\xi_{3}=\Lambda_{3} / M$. Then the leading contributions to each element of the Yukawa matrix is 


$$
\begin{aligned}
(U p)_{\bar{u}_{i} u_{j}} & =\left(\begin{array}{ccc}
s \xi_{1} \xi_{3} & 0 & \xi_{2}^{2} \\
\xi_{1}^{2} \xi_{2}^{2} \rho & \xi_{1} & \xi_{1}^{2} \rho \\
\xi_{2}^{2} & 0 & 1
\end{array}\right) \\
(\text { Down })_{\bar{d}_{i} d_{j}} & =\left(\begin{array}{ccc}
\xi_{1} \xi_{2}^{2} & \xi_{2} \xi_{3}+\bar{\rho} & \xi_{1} \rho \bar{\rho} \\
\xi_{1} \xi_{2} & \xi_{3} & 0 \\
\xi_{1} \xi_{2}^{2} & \xi_{2} \xi_{3}+\bar{\rho} & \xi_{1}
\end{array}\right) \\
(\text { Lepton })_{\bar{e}_{i} L_{j}} & =\left(\begin{array}{ccc}
\xi_{1} \xi_{2}^{2} & (s) \xi_{2} \xi_{3}+(a) \bar{\rho} & \xi_{1} \rho \bar{\rho} \\
\xi_{1} \xi_{2} & (s) \xi_{3} & 0 \\
\xi_{1} \xi_{2}^{2} & (s) \xi_{2} \xi_{3}+(a) \bar{\rho} & \xi_{1}
\end{array}\right)
\end{aligned}
$$

From the above mass matrices, a approximate texture zero structure [21.18] would be presented in the up quark mass matrix after determining the scale ratios. The down quark mass matrix and the lepton mass matrix are identical, except that the $(1,2),(2,2)$ and $(3,2)$ entries of the lepton mass matrix have different coefficients. These differences are due to the VEV patterns of $\langle\bar{\Sigma}\rangle$ and $\left\langle T_{3}\right\rangle$.

Before making further comments on the mass matrices, we would like to point out that if the introduced $Z_{3}$ symmetry is disabled in the model, then the forbidden terms such as $\left(\bar{\Sigma}^{2} / M^{2}+T_{2}^{2} T_{3}^{2} / M^{4}\right) 10_{3} 10_{2} H,\left(T_{1} T_{3}^{3} / M^{4}+T_{1}^{3} T_{2}^{2} / M^{5}\right) 10_{2} 10_{1} H$ and $\left(T_{1}^{2} T_{3} / M^{3}\right) 10_{3} \overline{5}_{2} \bar{H}$ will give additional contributions to $W_{u p}$ and $W_{\text {down-lepton }}$. We list these terms in the Appendix, Eq. (A.2) and (A.3). These new terms show the same hierarchy in powers of the small paramaters $\rho, \bar{\rho}$ and $\xi_{i}$. In other words, the mass hierarchy is merely determined by the gauge structure but not by the global discrete symmetry in the model.

Since this model cannot predict the coefficients for the coupling terms in superpotential, we assume these to be of order $\mathrm{O}(1)$ and ignore all coefficients in the above mass matrices. The zero entries in the up quark mass matrices are only approximate and could be replaced by those ignored subleading terms in Eq. (17). In fact, by the estimation made in later in this section, these "zeros" are such small numbers that they should be smaller than $10^{-11}$. Therefore, we can just ignore them in the later discussion. 
Although we do not know the coupling term coefficients, however, we can still extract some interesting points from Eq. (19 - 21). First, this model requires a low value of $\tan \beta$ because the top Yukawa coupling is much larger than the bottom Yukawa coupling.

We also observe that because of the VEV structures of $\langle\bar{\Sigma}\rangle$ and $\left\langle T_{3}\right\rangle$, the terms $\left(T_{2} T_{3} / M^{2}+\bar{\Sigma} / M\right) 10_{2} \overline{5}_{1} \bar{H}$ and $\left(T_{3} / M\right) 10_{2} \overline{5}_{2} \bar{H}$ have different contributions to the downquark mass matrix and the lepton mass matrix. It has long been a problem for $S U(5)$ grand unification that the mass relation $m_{l}=m_{d}$ at the GUT scale cannot be obeyed for all three generations. Georgi and Jarlskog [24,25], proposed a solution which has been used in models of SUSY $S O(10)$ grand unification [19]. Despite the successful experimental data fitting in their model, the low energy mass relation $m_{s} / m_{d}=25.15$ predicted in their model is two standard deviations away from the average value obtained by sum rule and chiral perturbation methods [20,18]. Our scheme does not give a definite prediction for the mass relations, but it does give some required extra freedom. For example, if the coefficient $s$ is taken to be 3 , then we obtain the GUT scale mass relations

$$
\begin{aligned}
& m_{\tau}=m_{b} \\
& m_{\mu} \approx 3 m_{s}
\end{aligned}
$$

These GUT mass relations could lead to acceptable $m_{b} / m_{\tau}$ and $m_{\mu} / m_{s}$ mass relations 18.21 at the weak scale.

A specific choice of the parameters that gives an acceptable representation of all of the experimental data on fermion masses is the following:

$$
\begin{aligned}
\frac{m_{c}}{m_{t}} & \sim \xi_{1} \sim O\left(10^{-2}\right), \\
\frac{m_{u}}{m_{c}} & \sim s \cdot \xi_{3} \sim O\left(10^{-2}\right) \\
\frac{m_{s}}{m_{b}} & \sim \frac{\xi_{3}}{\xi_{1}} \sim O\left(10^{-1}\right) \\
\frac{m_{e}}{m_{\mu}} & \sim \frac{\xi_{1} \xi_{2}^{2}}{s \xi_{3}}+\frac{a \xi_{1} \xi_{2}\left(\bar{\rho}+\xi_{2} \xi_{3}\right)}{\left(s \xi_{3}\right)^{2}} \sim O\left(10^{-2}\right) \\
\frac{m_{d}}{m_{s}} & \sim \frac{\xi_{1} \xi_{2}^{2}}{\xi_{3}}+\frac{\xi_{1} \xi_{2}\left(\bar{\rho}+\xi_{2} \xi_{3}\right)}{\xi_{3}^{2}} \sim O\left(10^{-1}\right)
\end{aligned}
$$


The above relations allow us to choose the scale ratios as

$$
\begin{aligned}
\xi_{1} & \sim \rho \sim \frac{1}{3} \times 10^{-2} \\
\xi_{2} & \sim 3 \times 10^{-2} \\
\xi_{3} & \sim \frac{1}{3} \times 10^{-3} \\
\bar{\rho} & \sim \frac{1}{2} \times 10^{-4} .
\end{aligned}
$$

From the $\mu$ value equation in (8), it can be easily checked that these values would give rise to a weak-scale $\mu$ value. Based on the given scale ratios, we can also estimate the CKM mixing angles $s_{12}, s_{23}$ and $s_{13}$ by

$$
s_{12}: s_{23}: s_{13} \sim \frac{\xi_{1} \xi_{2}}{\xi_{3}}: \frac{\bar{\rho}+\xi_{2} \xi_{3}}{\xi_{1}}: \xi_{2}^{2} \sim O\left(10^{-1}\right): O\left(10^{-2}\right): O\left(10^{-3}\right),
$$

which is consistent in order of magnitude with the experimental data. The GUT-group breaking scales are now determined to have the relation $\Lambda_{2}>\Lambda_{1}>\Lambda_{3}$. This confirms the breaking pattern described in Section 2.

According to the scale ratio estimations, there are approximate texture zero structures in the fermion mass matrices.

$$
\begin{aligned}
(U p)_{\bar{u}_{i} u_{j}} & =\left(\begin{array}{ccc}
s \xi_{1} \xi_{3} & 0 & \xi_{2}^{2} \\
0 & \xi_{1} & \xi_{1}^{2} \rho \\
\xi_{2}^{2} & 0 & 1
\end{array}\right) \\
(\text { Down })_{\bar{d}_{i} d_{j}} & =\left(\begin{array}{ccc}
\xi_{1} \xi_{2}^{2} & \xi_{2} \xi_{3}+\bar{\rho} \xi_{1} \rho \bar{\rho} \\
\xi_{1} \xi_{2} & \xi_{3} & 0 \\
\xi_{1} \xi_{2}^{2} & \xi_{2} \xi_{3}+\bar{\rho} & \xi_{1}
\end{array}\right) \\
(\text { Lepton })_{\bar{e}_{i} L_{j}} & =\left(\begin{array}{ccc}
\xi_{1} \xi_{2}^{2} & (s) \xi_{2} \xi_{3}+(a) \bar{\rho} & \xi_{1} \rho \bar{\rho} \\
\xi_{1} \xi_{2} & (s) \xi_{3} & 0 \\
\xi_{1} \xi_{2}^{2} & (s) \xi_{2} \xi_{3}+(a) \bar{\rho} & \xi_{1}
\end{array}\right)
\end{aligned}
$$

The zero entries are only approximate and represent values smaller than $10^{-10}$. Unlike the case in conventional SUSY flavor models [4, 21, 18, 11], these texture zeros are the natural 
outcome of the gauge structure as well as the scale ratios given in the model. In other words, they could arise without flavour symmetry.

In this section, we have estimated the possible scale ratio values needed to obtain acceptable fermion mass structures. The GUT gauge group $S U(5)_{1} \times S U(5)_{2} \times S U(5)_{3}$ would undergo a two-step breaking down to the SM group $S U(3)_{C} \times S U(2)_{L} \times U(1)_{Y}$. The SM gauge couplings unify at the scale of $10^{16} \mathrm{Gev}$ if we take the superheavy scale $M$ to be the reduced Planck scale. The Higgs triplets $H_{c}$ and $\bar{H}_{c}$ would obtain GUT scale masses of order of $10^{16} \mathrm{Gev}$ due to the superpotential term $\Sigma H \bar{H}$. Although we did not discuss the possible threshold effects [26] caused by those exotic Higgs fields as well as the heavy Higgs triplets, it is quite interesting that we find naturally a hierarchical pattern for the fermion mass matrices.

\section{PROTON DECAY}

We have already introduced a $Z_{2}^{\text {matter }}$ symmetry to disable all dangerous dimension three and four operators in Section two. However, since we find Higgs triplet masses of order $10^{16}$ $\mathrm{GeV}$, there is a danger that dimension five operators which violate baryon and lepton number could cause fast proton decay [27]. A dimension five operator in the superpotential could lead to proton decay if it has the form

$$
\frac{\lambda}{M^{*}} Q_{1} Q_{1} Q_{2} L_{i}
$$

Here $Q_{i}$ and $L_{i}$ represent the $i^{t h}$ generation of the quark and lepton multiplets respectively, $M^{*}$ represents some high scale, and $\lambda$ is the coupling constant. This operator leads to proton decay through the mode $p \longrightarrow K^{+} \bar{\nu}$. The current experiment data have already set the limit $\lambda / M^{*} \lesssim 10^{-24} \mathrm{GeV}^{-1}$ with the naturalness assumption that all squark/slepton masses are no larger than $1 \mathrm{TeV}$ 27,29]. In principle, operators of the form of Eq. (37) could arise from integrating out particles with GUT-scale masses or directly from the higher-dimension

operators in the original Lagrangian. In the Appendix, we analyze these higher-dimension 
operators and show that they are highly suppressed by powers of $1 / M$ due to the gauge structure as well as the $Z_{3}$ symmetry of the model. Therefore, the main contributions to proton decay in the model will come from heavy Higgsino exchange processes.

Since the VEV $\left\langle T_{1} T_{3}\right\rangle$ has vanishing contribution to color triplets, the potentially leading term $\left(T_{1} T_{3} / M^{2}\right) 10_{1} 10_{1} H$ cannot participate in the heavy Higgsino exchange processes. The same logic also applies to the terms such as $\left(T_{1} / M\right) 10_{2} 10_{2} H,\left(T_{1} T_{2} / M^{2}\right) 10_{1} \overline{5}_{2} \bar{H}$ and $\left(T_{1} T_{2}^{2} / M^{3}\right) 10_{1} \overline{5}_{1} \bar{H}$. Therefore, by taking the quark mixings into account, the leading terms in the superpotential that contribute to the dimension five operators in Eq. (37) are the following:

$$
\begin{array}{r}
10_{3} 10_{3} H \\
\left\{\frac{T_{2}^{2}}{M^{2}}\right\} 10_{1} 10_{3} H \\
\left\{\frac{T_{2}^{4}}{M^{4}}\right\} 10_{1} 10_{1} H \\
\left\{\frac{\bar{\Sigma}}{M}+\frac{T_{2} T_{3}}{M^{2}}\right\} 10_{2} \overline{5}_{1} \bar{H} \\
\left\{\frac{T_{3}}{M}\right\} 10_{2} \overline{5}_{2} \bar{H}
\end{array}
$$

From Eq.s (38 - 42), the leading dimension five operators that come from integrating out heavy Higgs triplets are shown in Fig. (11) and Fig. (2). We find that Fig. (1) should dominate the proton decay in the model with the decay mode $p \rightarrow K^{+} \bar{\nu}_{\mu}$. There are two contributions to Fig. (11), with coupling strengths

$$
\begin{aligned}
\frac{\lambda}{M^{*}} & \sim \frac{1}{M_{H_{c}}} \times \frac{\left\langle T_{2}^{4}\right\rangle}{M^{4}} \times \frac{\left\langle T_{3}\right\rangle}{M} \lesssim 10^{-25} \mathrm{Gev}^{-1} \\
\frac{\lambda}{M^{*}} & \sim \frac{\sin \theta_{13}}{M_{H_{c}}} \times \frac{\left\langle T_{2}^{2}\right\rangle}{M^{2}} \times \frac{\left\langle T_{3}\right\rangle}{M} \lesssim 10^{-25} \mathrm{Gev}^{-1} .
\end{aligned}
$$

In Eq. (43), the factor $\left\langle T_{2}^{4} / M^{4}\right\rangle$ comes from the next leading term $T_{2}^{4} 10_{1} 10_{1} H / M^{4}$ and the factor $\left\langle T_{3}\right\rangle / M$ comes from $\left(T_{3} / M\right) 10_{2} \overline{5}_{2} \bar{H}$ in superpotential. In Eq. (44), the factor $\left\langle T_{2}^{2} / M^{2}\right\rangle$ comes from the term $\left(T_{2}^{2} / M^{2}\right) 10_{1} 10_{3} H$ and $\sin \theta_{13}$ represents the mixing angle between the first and the third generation up-type quarks. The above coupling strength estimations show that the proton lifetime in the model should be no less than $10^{34}$ years. This result is 
about 100 times longer than the current experiment limit [28]. It is observed to the future experiment limit that could be set by SuperKamiokande.

Although there are uncertainties in determining the coefficients of the Yukawa coupling terms in the superpotential, however, the branching ratio between the $p \rightarrow K^{+} \bar{\nu}_{\mu}$ channel and the $p \rightarrow K^{+} \bar{\nu}_{e}$ could be definitely given by

$$
\frac{B R\left(p \rightarrow K^{+} \bar{\nu}_{e}\right)}{B R\left(p \rightarrow K^{+} \bar{\nu}_{\mu}\right)}=\left(\frac{\bar{\rho}+\xi_{2} \xi_{3}}{\xi_{3}}\right)^{2} \sim 10^{-2}
$$

This branching ratio prediction is generic to some SUSY models [30] that have the down quark mass generated by the seesaw mechanism. It is not clear to us how this prediction could be tested.

\section{CONCLUSION}

In this paper we have presented a supersymmetric GUT model based on the gauge group $S U(5)_{1} \times S U(5)_{2} \times S U(5)_{3}$. The Higgs fields and the matter fields are assigned to transform under the different $S U(5)$ groups in asymmetrical pattern. Exotic Higgs fields $\Sigma$, $\bar{\Sigma}, T_{1}, T_{2}$ and $T_{3}$ are needed to break the GUT gauge group down to the SM gauge group $S U(3)_{C} \times S U(2)_{L} \times U(1)_{Y}$, and also to relate matter fields which transform under different gauge $S U(5)$ 's. The discrete global symmetry $Z_{2}^{\text {matter }} \times Z_{3}$ is imposed at the reduced Planck scale in such a way that some dangerous terms in superpotential are disabled and a weakscale $\mu$ value for light Higgs doublets can be obtained. However, this discrete symmetry is the only flavour symmetry required in our scheme. The fermion mass hierarchy is a natural outcome of the gauge structure presented in this model. That is, it is the breaking of GUT gauge group but not the breaking of flavour symmetry that generates the fermion mass hierarchy in our model. In Section 3, we have shown that realistic fermion mass matrices can be the result of this mechanism. The fermion mass relations and the CKM angles are estimated to be consistent with measured experiment data at low energy. The exotic Higgs fields also play important roles in predicting realistic down-quark and lepton mass relations. 
The fields $\bar{\Sigma}$ and $T_{3}$ allow us to obtain the Georgi-Jarlskog relation between the leptons and down quark masses, and also more general relations that may be required by experiment.

This model does not forbid the dimension five operators that could result in nucleon decays. In fact, there are allowed tree-level dimension five operators in the superpotential. However, these tree-level terms are suppressed by powers of the superheavy scale $M$ and thus are not important in discussing the proton decay. The proton decay in the model is mainly due to Higgsino-exchange processes. The dominant mode of proton decay in the model is the process $p \rightarrow K^{+} \bar{\nu}_{\mu}$, the same dominant mode as in minimal $\operatorname{SUSY} \operatorname{SU}(5)$ model. Due to the VEV pattern of the field $T_{1}$, the leading term $\left(T_{1} T_{3} / M^{2}\right) 10_{1} 10_{1} H$ terms in the superpotential does not participate in the heavy Higgs triplet exchange process and thus gives zero contribution to the proton decay. The next leading order contributions of proton decay come from the term $\left(T_{2}^{4} / M^{4}\right) 10_{1} 10_{1} H$ and quark mixing effects, which are more suppressed than the leading order term $\left(T_{1} T_{3} / M^{2}\right) 10_{1} 10_{1} H$. Therefore, proton decay in this model is highly sensistive to changes of the scale ratio $\left\langle T_{2}\right\rangle / M$. The proton lifetime

is estimated to be larger than $10^{34}$ years, depending on the exact $\left\langle T_{2}\right\rangle / M$ value and the unknown coefficients of coupling terms in superpotential.

Models with product SU(5) groups were originally introduced with motivations from string theory. Our model shows that this structure may be interesting in its own right as a possible explanation of the fermion mass spectra.

\section{APPENDIX A:}

If the $Z_{3}$ symmetry is not introduced to the model, then all possible operators bilinear in $H$ and $\bar{H}$ that are up to the dimension 10 level are given as follows:

$$
\begin{aligned}
W_{H \bar{H}} & =\Sigma H \bar{H}\left\{1+\frac{\Sigma \bar{\Sigma}}{M^{2}}+\frac{T_{1} \bar{\Sigma}}{M^{2}}+\frac{T_{1} T_{2} T_{3}}{M^{3}}+\frac{\Sigma T_{2} T_{3}}{M^{3}}+\frac{(\Sigma \bar{\Sigma})^{2}}{M^{4}}+\frac{\left(T_{1} \bar{\Sigma}\right)^{2}}{M^{4}}\right. \\
& +\frac{(\Sigma \bar{\Sigma})\left(T_{1} \bar{\Sigma}\right)}{M^{4}}+\frac{1}{M^{5}}\left[(\Sigma \bar{\Sigma})\left(\Sigma T_{2} T_{3}+T_{1} T_{2} T_{3}\right)+\left(T_{1} \bar{\Sigma}\right)\left(\Sigma T_{2} T_{3}+T_{1} T_{2} T_{3}\right)\right. \\
& \left.+\sum_{k=0}^{5} \frac{1}{M^{5-k}} \bar{\Sigma}^{k}\left(T_{2} T_{3}\right)^{5-k}+\sum_{k=0}^{5} \Sigma^{k} T_{1}^{5-k}+T_{2}^{5}+T_{3}^{5}\right]
\end{aligned}
$$




$$
\begin{aligned}
& \left.+\frac{1}{M^{6}}\left[\left(T_{1} T_{2} T_{3}\right)^{2}+\left(T_{1} T_{2} T_{3}\right)\left(\Sigma T_{2} T_{3}\right)+\left(\Sigma T_{2} T_{3}\right)^{2}+\sum_{k=0}^{3}\left(T_{1} \bar{\Sigma}\right)^{k}(\Sigma \bar{\Sigma})^{3-k}\right]\right\} \\
& +T_{1} H \bar{H}\left\{1+\frac{\Sigma \bar{\Sigma}}{M^{2}}+\frac{T_{1} \bar{\Sigma}}{M^{2}}+\frac{T_{1} T_{2} T_{3}}{M^{3}}+\frac{\Sigma T_{2} T_{3}}{M^{3}}+\frac{(\Sigma \bar{\Sigma})^{2}}{M^{4}}+\frac{\left(T_{1} \bar{\Sigma}\right)^{2}}{M^{4}}\right. \\
& +\frac{(\Sigma \bar{\Sigma})\left(T_{1} \bar{\Sigma}\right)}{M^{4}}+\frac{1}{M^{5}}\left[(\Sigma \bar{\Sigma})\left(\Sigma T_{2} T_{3}+T_{1} T_{2} T_{3}\right)+\left(T_{1} \bar{\Sigma}\right)\left(\Sigma T_{2} T_{3}+T_{1} T_{2} T_{3}\right)\right. \\
& \left.+\sum_{k=0}^{5} \frac{1}{M^{5-k}} \bar{\Sigma}^{k}\left(T_{2} T_{3}\right)^{5-k}+\sum_{k=0}^{5} \Sigma^{k} T_{1}^{5-k}+T_{2}^{5}+T_{3}^{5}\right]+\frac{1}{M^{6}}\left[\left(T_{1} T_{2} T_{3}\right)^{2}\right. \\
& \left.\left.+\left(T_{1} T_{2} T_{3}\right)\left(\Sigma T_{2} T_{3}\right)+\left(\Sigma T_{2} T_{3}\right)^{2}+\sum_{k=0}^{3}\left(T_{1} \bar{\Sigma}\right)^{k}(\Sigma \bar{\Sigma})^{3-k}\right]\right\}
\end{aligned}
$$

The leading Yukawa coupling terms that give masses to fermions are also listed below:

$$
\begin{aligned}
W_{u p} & =H\left\{10_{3} 10_{3}+\frac{T_{1}}{M} 10_{2} 10_{2}+\frac{T_{2}^{2}}{M^{2}} 10_{3} 10_{1}+\frac{T_{1} T_{3}}{M^{2}} 10_{1} 10_{1}+\right. \\
& +10_{3} 10_{2}\left[\frac{\bar{\Sigma}^{2}}{M^{2}}+\sum_{k=0}^{3} \frac{\Sigma^{3-k} T_{1}^{k}}{M^{3}}+\frac{\left(T_{2} T_{3}\right)^{2}}{M^{4}}\right] \\
& +10_{2} 10_{1}\left[\frac{T_{1} T_{3}^{3}}{M^{4}}+\frac{T_{2}^{2} \bar{\Sigma}^{2}}{M^{4}}+\sum_{k=0}^{3} \frac{T_{1}^{k} T_{2}^{2} \Sigma^{3-k}}{M^{5}}\right] \\
& +\cdots\} \\
W_{\text {down-lepton }}= & \bar{H}\left\{\frac{T_{1}}{M} 10_{3} \overline{5}_{3}+\frac{T_{1}^{2} T_{3}+\Sigma^{2} T_{3}}{M^{3}} 10_{3} \overline{5}_{2}+\frac{T_{3} T_{2}+\Sigma}{M^{2}} 10_{2} \overline{5}_{3}+\frac{T_{3}}{M} 10_{2} \overline{5}_{2}\right. \\
+ & 10_{3} \overline{5}_{1}\left(\frac { T _ { 1 } } { M ^ { 2 } } \left[\frac{T_{1} \bar{\Sigma}+\Sigma \bar{\Sigma}}{M^{2}}+\frac{T_{1} T_{2} T_{3}+\Sigma T_{2} T_{3}}{M^{3}}\right.\right. \\
+ & \left.\frac{\left(T_{1} \bar{\Sigma}\right)^{2}+\left(T_{1} \bar{\Sigma}\right)(\Sigma \bar{\Sigma})+(\Sigma \bar{\Sigma})^{2}}{M^{4}}\right] \\
& \left.+\frac{\bar{\Sigma}^{4}}{M^{4}}\right)+\left(\frac{T_{3} T_{2}}{M^{2}}+\frac{\bar{\Sigma}}{M}\right) 10_{2} \overline{5}_{1}+\frac{T_{1} T_{2}^{2}}{M^{3}} 10_{1} \overline{5}_{3} \\
& \left.+\frac{T_{2} T_{1}}{M^{2}} 10_{1} \overline{5}_{2}+\frac{T_{1} T_{2}^{2}}{M^{3}} 10_{1} \overline{5}_{1}+\cdots\right\} .
\end{aligned}
$$

From (A.2) and (A.3), a hierarchical and texure of fermion masses is still present in the model even without introducing the $Z_{3}$ symmetry. This can be seen by the following fermion mass matrices.

$$
(\mathrm{Up})_{\bar{u}_{i} u_{j}}=\left(\begin{array}{ccc}
(s) \xi_{1} \xi_{3} & (s) \xi_{1} \xi_{3}^{3}+\xi_{2}^{2} \bar{\rho}^{2}+\xi_{1} \xi_{2}^{2} \rho^{2} & \xi_{2}^{2} \\
\left(s^{2}\right) \xi_{1} \xi_{3}^{3}+\xi_{1}^{2} \xi_{2}^{2} \rho & \xi_{1} & (a) \bar{\rho}^{2}+\xi_{1} \rho^{2}+\xi_{1}^{2} \rho \\
\xi_{2}^{2} & \bar{\rho}^{2}+\xi_{1} \rho^{2}+\xi_{1}^{2} \rho & 1
\end{array}\right)
$$




$$
\begin{aligned}
\text { (Down) })_{\bar{d}_{i} u_{j}} & =\left(\begin{array}{ccc}
\xi_{1} \xi_{2}^{2} & \xi_{2} \xi_{3}+\bar{\rho} & \xi_{1}^{2} \bar{\rho}+\xi_{1} \rho \bar{\rho} \\
\xi_{1} \xi_{2} & \xi_{3} & \rho^{2} \xi_{3} \\
\xi_{1} \xi_{2}^{2} & \xi_{2} \xi_{3}+\bar{\rho} & \xi_{1}
\end{array}\right) \\
\text { (Lepton })_{\bar{e}_{i} L_{j}} & =\left(\begin{array}{ccc}
\xi_{1} \xi_{2}^{2} & (s) \xi_{2} \xi_{3}+(a) \bar{\rho} & \xi_{1}^{2} \bar{\rho}+\xi_{1} \rho \bar{\rho} \\
\xi_{1} \xi_{2} & (s) \xi_{3} & \xi_{1}^{2} \xi_{3} \\
\xi_{1} \xi_{2}^{2} & (s) \xi_{2} \xi_{3}+(a) \bar{\rho} & \xi_{1}
\end{array}\right)
\end{aligned}
$$

From the above matrices, the approximate texture zero structures will be present as a result of the gauge structure of the model. The up quark mass matrix (A.4) becomes slightly asymmetrical due to the VEV structures given in Eq. (5) and the gauge structure of this model. The coefficients $(s),\left(s^{2}\right)$ and $(a)$ in the above matrices indicate the additional factors that come from the constants $s$ and $a$ in the VEV $\left\langle T_{3}\right\rangle$ and $\langle\bar{\Sigma}\rangle$. Alltogether, these make the down quark and the lepton mass matrices different from each other even though they arise from the same superpotential $W_{\text {down-lepton }}$.

Without imposing $Z_{3}$ symmetry onto this model, if we forbid possible dangerous dimension three and four operators by introducing $Z_{2}^{\text {matter }}$ symmetry, there could still exist some leading tree level operators that would mediate proton decay.

$$
\begin{array}{r}
\left(1+\frac{\Sigma \bar{\Sigma}}{M^{2}}+\frac{T_{1} \bar{\Sigma}}{M^{2}}+\cdots\right) \frac{T_{3}^{2}}{M^{3}} 10_{1} 10_{1} 10_{2} \overline{5}_{2}, \\
\left(1+\frac{\Sigma \bar{\Sigma}}{M^{2}}+\frac{T_{1} \bar{\Sigma}}{M^{2}}+\cdots\right) \frac{\left(\bar{\Sigma} T_{2}\right)^{3}}{M^{7}} 10_{1} 10_{1} 10_{2} \overline{5}_{2}, \\
\left(1+\frac{\Sigma \bar{\Sigma}}{M^{2}}+\frac{T_{1} \bar{\Sigma}}{M^{2}}+\cdots\right) \frac{T_{3} \bar{\Sigma}}{M^{3}} 10_{1} 10_{1} 10_{2} \overline{5}_{1}, \\
\left(1+\frac{\Sigma \bar{\Sigma}}{M^{2}}+\frac{T_{1} \bar{\Sigma}}{M^{2}}+\cdots\right) \frac{T_{2}^{4} \bar{\Sigma}^{2}}{M^{7}} 10_{1} 10_{1} 10_{2} \overline{5}_{1}, \\
\left(1+\frac{\Sigma \bar{\Sigma}}{M^{2}}+\frac{T_{1} \bar{\Sigma}}{M^{2}}+\cdots\right) \frac{\Sigma T_{2}}{M^{3}} 10_{1} 10_{2} 10_{2} \overline{5}_{2}, \\
\left(1+\frac{\Sigma \bar{\Sigma}}{M^{2}}+\frac{T_{1} \bar{\Sigma}}{M^{2}}+\cdots\right) \frac{T_{2}^{3} T_{1}^{2} \Sigma}{M^{7}} 10_{1} 10_{1} 10_{2} \overline{5}_{2} \\
\left(1+\frac{\Sigma \bar{\Sigma}}{M^{2}}+\frac{T_{1} \bar{\Sigma}}{M^{2}}+\cdots\right) \frac{\Sigma T_{3}}{M^{3}} 10_{1} 10_{1} 10_{3} \overline{5}_{1} \\
\left(1+\frac{\Sigma \bar{\Sigma}}{M^{2}}+\frac{T_{1} \bar{\Sigma}}{M^{2}}+\cdots\right) \frac{T_{2}^{3}}{M^{4}} 10_{1} 10_{1} 10_{3} \overline{5}_{2} \\
\left(1+\frac{\Sigma \bar{\Sigma}}{M^{2}}+\frac{T_{1} \bar{\Sigma}}{M^{2}}+\cdots\right) \frac{\bar{\Sigma}^{2}}{M^{3}} 10_{2} 10_{3} 10_{3} \overline{5}_{1}
\end{array}
$$




$$
\begin{array}{r}
\left(1+\frac{\Sigma \bar{\Sigma}}{M^{2}}+\frac{T_{1} \bar{\Sigma}}{M^{2}}+\cdots\right) \frac{T_{3} \bar{\Sigma}}{M^{3}} 10_{2} 10_{3} 10_{3} \overline{5}_{2} \\
\left(1+\frac{\Sigma \bar{\Sigma}}{M^{2}}+\frac{T_{1} \bar{\Sigma}}{M^{2}}+\cdots\right) \frac{\Sigma}{M^{2}} 10_{2} 10_{2} 10_{3} \overline{5}_{1} \\
\left(1+\frac{\Sigma \bar{\Sigma}}{M^{2}}+\frac{T_{1} \bar{\Sigma}}{M^{2}}+\cdots\right) \frac{\Sigma T_{3} T_{1}}{M^{4}} 10_{2} 10_{2} 10_{3} \overline{5}_{2}
\end{array}
$$

The above non-renormalizable operators, if they exist in our model, would give effective dimension five operators that violate baryon and lepton numbers. By the scale ratios given in Section 3, we find the largest two coupling strengths in the list to come from Eq. (A.11) and (A.17)

$$
\begin{aligned}
\sin \theta_{23} \sin \theta_{c} \frac{\langle\Sigma\rangle}{M^{2}} \sim \sin \theta_{c} \frac{\left\langle\Sigma T_{2}\right\rangle}{M^{3}} \sim \frac{10^{-5}}{M} & \sim O\left(10^{-23}\right) \mathrm{Gev}^{-1} \\
& >O\left(10^{-24}\right) \mathrm{GeV}^{-1}
\end{aligned}
$$

where the superheavy scale $M$ is taken to be the reduced Planck scale. This result would predict a proton lifetime which is about $10^{2}$ times shorter than the current experiment limit. Fortunately, if the $Z_{3}$ symmetry is introduced, some of the tree-level terms in Eq.s A.7 A.18) are forbidden. We are thus left with the leading tree-level terms of Eq. (A.7), (A.9) and (A.16).

$$
\begin{array}{rlrl}
\frac{\left(T_{1} \bar{\Sigma}\right)}{M^{2}} \frac{T_{3}^{2}}{M^{3}} 10_{1} 10_{1} 10_{2} \overline{5}_{2}, & \frac{\lambda}{M^{*}} \sim \frac{\left\langle\left(T_{1} \bar{\Sigma}\right) T_{3}^{2}\right\rangle}{M^{5}} \sim \frac{10^{-13}}{M} \\
\frac{\left(T_{1} \bar{\Sigma}\right)}{M^{2}} \frac{T_{3} \bar{\Sigma}}{M^{3}} 10_{1} 10_{1} 10_{2} \overline{5}_{1}, & \frac{\lambda}{M^{*}} \sim \frac{\left\langle T_{3} \bar{\Sigma}\left(T_{1} \bar{\Sigma}\right)\right\rangle}{M^{4}} \sim \frac{10^{-14}}{M} \\
\frac{T_{3} \bar{\Sigma}}{M^{3}} 10_{2} 10_{3} 10_{3} \overline{5}_{2}, & \frac{\lambda}{M^{*}} \sim \sin \theta_{13}^{2} \frac{\left\langle T_{3} \bar{\Sigma}\right\rangle}{M^{3}} & \sim \frac{10^{-13}}{M} .
\end{array}
$$

These terms are much less important than the Higgsino-exchange processes in Eq. (43) and (44). Therefore, they could just be ignored in discussing the proton decay in this model.

\section{Acknowledgments}

We thank M.E. Peskin, Y. Shirman and N. Arkani-Hamed for useful discussions, and especially thank M. E. Peskin for reading the manuscript. This work is supported by Department of Energy contract DE-AC03-76SF00515. 


\section{REFERENCES}

[1] M. E. Peskin, "Beyond the Standard Model" hep-ph/9705479.

[2] H. E. Haber, G. L. Kane,Phys. Rept. 117 (1985)75.

S. Dawson, "The MSSM and Why It Works" hep-ph/9712464.

[3] S. Dimopoulos and H. Georgi, Nucl. Phys. B193(1981)150.

S. Dimopoulos, S. Raby and F. Wilczek,Phys. Rev. D24(1981) 1681.

P. Langacker and M. Luo, Phys. Rev. D44(1991)514.

J. Ellis, S. Kelley and D. Nanopoulos, Phys. Lett. B260(1991) 447.

P. Langacker and N. Polonsky, Phys. Rev. D47(1993)4028.

[4] R. Barbieri, G. Dvali and L. J. Hall, Phys. Lett., B377(1996)76-82.

Z. Berezhiani, Nucl. Phys. Proc. Suppl. 52A(1997)153-158.

D. B. Kaplan and M. Schmaltz, Phys. Rev. D49(1994)3741-3750.

E. Dudas, S. Pokorski and C. A. Savoy, Phys. Lett. B356(1995) 45-55.

[5] J. Ellis, D. V. Nanopoulos, Phys. Lett. B110 (1982)44.

A. Masiero and L. Silverstrini, hep-ph/9711401.

M. Misiak, S. Pokorski and J. Rosiek, hep-ph/9703442.

C. A. Savoy, "FCNC in SUSY Theories", hep-ph/9601225.

[6] R.N. Mohapatra,hep-ph/9801235.

R. Barbieri, G. Dvali, A. Strumia, Phys.Lett. B333(1994)79-82.

[7] Z. Berezhiani, Nucl.Phys.Proc.Suppl. 52A (1997)153-158.

L. J. Hall, S. Raby, Phys.Rev. D51(1995)6524-6531.

R. Barbieri, L. J. Hall, S. Raby, A. Romanino, Nucl.Phys. B493(1997)3-26.

D. B.Kaplan, M. Schmaltz, Phys.Rev. D49(1994)3741-3750.

R. Barbieri,G. Dvali, L.J. Hall, Phys.Lett. B377(1996)76-82.

[8] L, Lykken, T. Montroy and S. Willenbrock, hep-ph/9710492. 
D. G. Lee and R. N. Mohapatra, Phys. Rev. D51(1995)1353.

[9] K. S. Babu and S. M. Barr, Phys. Rev. D56(1997) 2614-2631.

[10] K.R. Dienes, Phys. Rept. 287(1997)447-525.

[11] K.S. Babu and S. Barr, Phys. Rev. D48(1993) 5354.

Z. Berezhiani, hep-ph/9602325.

[12] C.H. Albright, S.M. Barr, hep-ph/9712488.

S. M. Barr and S. Raby, Phys. Rev. Lett. 79(1997)4748-4751.

[13] R. N. Mohapatra, Phys. Lett. B379(1996)115-120.

R. Barbieri, G. Dvali and A. Strumia, Phys. Lett., B333(1994) 79.

A. Maslikov, I. Naumov and G. Volkov, Phys. Lett. B409 (1997)160-172.

[14] R. Barbieri, G. Dvali, A. Strumia, Nucl.Phys. B435(1995)102-114.

[15] E. Witten, Phys. Lett., B105 (1981)267.

D.V. Nanopoulos and K. Tamvakis, Phys. Lett., B113 (1982)151.

S. Dimopoulos and H. Georgi, Phys. Lett., B117 (1982)287.

S. M. Barr, Phys. Rev. D57(1998)190.

[16] J. Polchinski and L. Susskind, Phys. Rev., D26(1982)3661.

H. P. Nilles, M.Srednicki and D. Wyler, Phys Lett., B124 (1983)337.

A. B. Lahanas, Phys. Lett., B124(1983)341.

[17] S. Raby, OHSTPY-HEP-T-95-024, hep-ph/9501349.

[18] G.Anderson, S.Raby, S.Dimopoulos, L.J.Hall and G.D.Starkman, Phys.Rev. D49(1994)3660-3690.

K. S. Babu, S. M. Barr, Phys.Rev. D56(1997)2614-2631.

[19] S. Dimopoulos, L.J.Hall and S. Raby, Phys. Rev. D45(1992)4192-4200. 
[20] T. Bhattacharya and R. Gupta, hep-lat/9710095.

[21] P. Ramond, R. G. Roberts, G. G. Ross, Nucl.Phys. B406(1993)19-42.

S. Dimopoulos, L. J. Hall, S. Bary, Phys.Rev. D45(1992) 4192-4200.

M. E. Gomez, K. Tamvakis, hep-ph/9801348.

[22] S. M. Barr, Phys. Rev. D55(1997) 6775-6779.

[23] Z.Berezhiani, Z. Tavartkiladze, Phys.Lett. B396(1997)150-160.

Z.Berezhiani, Z. Tavartkiladze, Phys.Lett. B409(1997) 220-228.

[24] H. Georgi, C. Jarlskog, Phys. Lett. B86 (1979)297.

[25] K. S. Babu, R. N. Mohapatra, Phys. Rev. Lett. 74(1995)2418-2421.

[26] J. Hisano, H. Murayama and T. Yanagida, Nucl. Phys. B402(1993)46.

R. Barbieri and L. J. Hall, Phys. Rev. Lett., 68(1992)752.

[27] S. Weinberg, Phys. Rev., D26(1982)287.

N. Sakai and T. Yanagida, Nucl. Phys. , B197(1982)533.

J. Hisano, H. Murayama, T. Yanagida, Nucl. Phys. B402 (1993)46.

H. Murayama, D. B. Kaplan, Phys. Lett. B336(1994)221-228.

K.S. Babu and S. M. Barr, Phys. Lett. B381(1996)137.

[28] R. M. Barnette et al., "Review of Particle Physics" , Phys. Rev. D54(1996)1.

[29] H. Murayama, hep-ph/9801331.

[30] V. Lucas and S. Raby, Phys.Rev. D55(1997) 6986-7009 


\section{FIGURES}

FIG. 1. Dimension five operators produced by integrating out heavy Higgs triplets. These two operators both dominate the proton decay due to the quark mixing.

FIG. 2. Dimension five operators produced by integrating out heavy Higgs triplets. These two operators both contribute to the proton decay due to the quark mixing. 


\section{TABLES}

TABLE I. The Field content of the model.

\begin{tabular}{lccc}
\hline \hline & $S U(5)_{1}$ & $S U(5)_{2}$ & $S U(5)_{3}$ \\
\hline$\Sigma$ & & 5 & $\overline{5}$ \\
$\bar{\Sigma}$ & & $\overline{5}$ & 5 \\
$T_{1}$ & $\overline{5}$ & 5 & $\overline{5}$ \\
$T_{2}$ & 5 & 5 \\
$T_{3}$ & $\overline{5}$ & 10 \\
\hline $10_{3}$ & & & \\
$10_{2}$ & & 10 & \\
$10_{1}$ & & & $\overline{5}$ \\
$\overline{5}_{3}$ & 10 & & \\
$\overline{5}_{2}$ & & & \\
$\overline{5}_{1}$ & $\overline{5}$ & & 5 \\
$\mathrm{H}$ & & & \\
$\bar{H}$ & & & \\
\hline \hline
\end{tabular}

